\title{
Quaternionic Hyperbolic Function Theory
}

\section{Eriksson, Sirkka-Liisa}

Springer International Publishing 2019

Eriksson , S-L \& Orelma , H 2019 , Quaternionic Hyperbolic Function Theory . in Topics in Clifford Analysis : Special Volume in Honor of Wolfgang Sprößig . Trends in Mathematics, Springer International Publishing , pp. 25-52 . https://doi.org/10.1007/978-3-030-23854-4_2

http://hdl.handle.net/10138/320370

https://doi.org/10.1007/978-3-030-23854-4_2

unspecified

acceptedVersion

Downloaded from Helda, University of Helsinki institutional repository.

This is an electronic reprint of the original article.

This reprint may differ from the original in pagination and typographic detail.

Please cite the original version. 


\title{
Quaternionic Hyperbolic Function Theory
}

\author{
Sirkka-Liisa Eriksson and Heikki Orelma
}

Abstract We are studying hyperbolic function theory in the skew-field of quaternions. This theory is connected to $k$-hyperbolic harmonic functions that are harmonic with respect to the hyperbolic Riemannian metric

$$
d s_{k}^{2}=\frac{d x_{0}^{2}+d x_{1}^{2}+d x_{2}^{2}+d x_{3}^{2}}{x_{3}^{k}}
$$

in the upper half space $\mathbb{R}_{+}^{4}=\left\{\left(x_{0}, x_{1}, x_{2}, x_{3}\right) \in \mathbb{R}^{4}: x_{3}>0\right\}$. In the case $k=2$, the metric is the hyperbolic metric of the Poincaré upper half-space. Hempfling and Leutwiler started to study this case and noticed that the quaternionic power function $x^{m}(m \in \mathbb{Z})$, is a conjugate gradient of a 2-hyperbolic harmonic function. They researched polynomial solutions. We find fundamental $k$-hyperbolic harmonic functions depending only on the hyperbolic distance and $x_{3}$. Using these functions we are able to verify a Cauchy type integral formula. Earlier these results have been verified for quaternionic functions depending only on reduced variables $\left(x_{0}, x_{1}, x_{2}\right)$. Our functions are depending on four variables.

\section{Introduction}

We study hyperbolic function theory in the skew- field of quaternions, denoted by $\mathbb{H}$. This theory was initiated by Thomas Hempfling and Heinz Leutwiler in [15]. They studied quaternion valued twice continuous differentiable functions $f(x)$ defined in

Sirkka-Liisa Eriksson

Department of Mathematics and Statistics, University of Helsinki, P.O.Box 68, FI-00014 Helsinki, e-mail: sirkka-liisa.eriksson@ helsinki.fi

Heikki Orelma

Laboratory of Civil Engineering, Tampere University of Technology, Korkeakoulunkatu 10, 33720

Tampere, Finland e-mail: heikki.orelma@tut.fi 
the full space $\mathbb{R}^{4}$ satisfying the following modified Cauchy-Riemann system

$$
\begin{gathered}
x_{3}\left(\frac{\partial f_{0}}{\partial x_{0}}-\frac{\partial f_{1}}{\partial x_{1}}-\frac{\partial f_{2}}{\partial x_{2}}-\frac{\partial f_{3}}{\partial x_{3}}\right)+2 f_{3}=0, \\
\frac{\partial f_{0}}{\partial x_{i}}=-\frac{\partial f_{i}}{\partial x_{0}} \text { for all } i=1,2,3, \\
\frac{\partial f_{i}}{\partial x_{j}}=\frac{\partial f_{j}}{\partial x_{i}} \text { for all } i, j=1,2,3 .
\end{gathered}
$$

In [17] Leutwiler noticed that the power function $x^{m}$, where $m \in \mathbb{Z}$, calculated using quaternions, is a conjugate gradient of a hyperbolic harmonic function $h$ which satisfies the equation

$$
\Delta_{2} h=x_{3}^{2} \Delta h-2 x_{3} \frac{\partial h}{\partial x_{3}}=0
$$

where as usual

$$
\Delta h=\frac{\partial^{2} h}{\partial x_{0}^{2}}+\frac{\partial^{2} h}{\partial x_{1}^{2}}+\frac{\partial^{2} h}{\partial x_{2}^{2}}+\frac{\partial^{2} h}{\partial x_{3}^{2}}
$$

The operator $\Delta_{2}$ is the hyperbolic Laplace-Beltrami operator with respect to the Poincaré hyperbolic metric

$$
d s^{2}=\frac{d x_{0}^{2}+d x_{1}^{2}+d x_{2}^{2}+d x_{3}^{2}}{x_{3}^{2}} .
$$

These functions are called conjugate gradients of real hyperbolic harmonic functions.

Leutwiler and the first author in [7] studied the total Clifford algebra valued functions, called hypermonogenic functions. Their Cauchy-type formula was proved in [6] and the key ideas are the relations between $k$ and $-k$-hypermonogenic functions, introduced in [3]. An introduction to the theory is given in [18] and in more recent paper [8].

In this paper, we verify the Cauchy type theorems for quaternionic valued fuctions called $k$-hyperregular. Our Cauchy type theorems are not directly following from the theory of quaternionic valued hypermonogenic functions, which are depending only on three variables. Our functions are depending on four variables and $k$ is an arbitrary real coefficient. However, it is possible to deduce some results from the theory of paravector valued $k$-hypermonogenic funcions (see [9]) which domain of the definition is an open subset of $\mathbb{R}^{4}$ and the values are in the Clifford algebra $\mathscr{C} \ell_{0,3}$. These methods are rather complicated in case of quaternions and we prefer the direct methods. 


\section{Preliminaries}

The space of quaternions $\mathbb{H}$ is four dimensional associative division algebra over reals with an identity 1 and generated by the elements $\mathbf{1}, e_{1}, e_{2}$ and $e_{3}$ satisfying the relations

$$
e_{3}=e_{1} e_{2}
$$

and

$$
e_{i} e_{j}+e_{j} e_{i}=-2 \delta_{i j} \mathbf{1}
$$

where $\delta_{i j}$ is the usual Kronecker delta. The elements $\alpha \mathbf{1}$ and $\alpha$ may be identified.

We denote the coefficients of the components of a quaternion $x$ with respect to the base $\left\{1, e_{0}, e_{1}, e_{2}\right\}$ by $x_{0}, x_{1}, x_{2}$ and $x_{3}$, that is

$$
x=x_{0}+x_{1} e_{1}+x_{2} e_{2}+x_{3} e_{3}
$$

where $x_{0}, x_{1}, x_{2}$ and $x_{3}$ are real numbers. The spaces $\mathbb{R}^{4}$ and $\mathbb{H}$ may be identified as vector spaces.

We denote the upper half space by

$$
\mathbb{H}_{+}=\left\{x \mid x_{i} \in \mathbb{R}, i=0,1,2,3 \text { and } x_{3}>0\right\}
$$

and the lower half space by

$$
\mathbb{H}_{-}=\left\{x \mid x_{i} \in \mathbb{R} i=0,1,2,3 \text { and } x_{3}<0\right\} .
$$

The hyperbolic distance $d_{h}(x, a)$ between the points $x$ and $a$ in $\mathbb{H}_{+}$may be computed from the formula $d_{h}(x, a)=\operatorname{arcosh} \lambda(\mathrm{x}, \mathrm{a})$, where

$$
\begin{aligned}
\lambda(x, a) & =\frac{\left(x_{0}-a_{0}\right)^{2}+\left(x_{1}-a_{1}\right)^{2}+\left(x_{2}-a_{2}\right)^{2}+x_{3}^{2}+a_{3}^{2}}{2 x_{3} a_{3}} \\
& =\frac{\|x-a\|^{2}+\left\|x-a^{*}\right\|^{2}}{4 x_{3} a_{3}} \\
& =\frac{\|x-a\|^{2}}{2 x_{3} a_{3}}+1=\frac{\left\|x-a^{*}\right\|^{2}}{2 x_{3} a_{3}}-1,
\end{aligned}
$$

putting $a^{*}=a_{0}+a_{1} e_{1}+a_{2} e_{2}-a_{3} e_{3}$ and the distance

$$
\|x-a\|=\sqrt{\left(x_{0}-a_{0}\right)^{2}+\left(x_{1}-a_{1}\right)^{2}+\left(x_{2}-a_{2}\right)^{2}+\left(x_{3}-a_{3}\right)^{2}}
$$

is the usual Euclidean distance (see the proof for example in [18]). Similarly, we may compute the hyperbolic distance between the points $x$ and $a$ in $\mathbb{H}_{-}$. Notice that if both $x$ and $a$ belong to $\mathbb{H}_{+}$or in $\mathbb{H}_{-}$then

$$
d_{h}(x, a)=d_{h}\left(x^{*}, a^{*}\right) .
$$


We recall the following simple calculation rules

$$
\begin{aligned}
\|x-a\|^{2} & =2 x_{3} a_{3}(\lambda(x, a)-1), \\
\left\|x-a^{*}\right\|^{2} & =2 x_{3} a_{3}(\lambda(x, a)+1), \\
\frac{\|x-a\|^{2}}{\left\|x-a^{*}\right\|^{2}} & =\frac{\lambda(x, a)-1}{\lambda(x, a)+1}=\tanh ^{2}\left(\frac{d_{h}(x, a)}{2}\right) .
\end{aligned}
$$

We remind that hyperbolic balls are also Euclidean balls with a shifted center given by the next result.

Proposition 1. The hyperbolic ball $B_{h}\left(a, r_{h}\right)$ with the hyperbolic center a in $\mathbb{H}_{+}$and the radius $r_{h}$ is the same as the Euclidean ball with the Euclidean center

$$
c_{a}\left(r_{h}\right)=a_{0}+a_{1} e_{1}+a_{2} e_{2}+a_{3} \cosh r_{h} e_{3}
$$

and the Euclidean radius $r_{e}=a_{3} \sinh r_{h}$. Conversely, if $b=\left(b_{0}, b_{1}, b_{2}, b_{3}\right)$ is a point in $\mathbb{H}_{+}$and $r_{e}<b_{3}$ then the Euclidean ball $B_{e}\left(b, r_{e}\right)$ is the same as the hyperbolic ball with the hyperbolic radius

$$
r_{h}=\operatorname{artanh}\left(\frac{r_{e}}{b_{3}}\right)
$$

and the hyperbolic center

$$
a=\left(b_{0}, b_{1}, b_{2}, \frac{b_{3}}{\cosh r_{h}}\right) .
$$

Corollary 1. The hyperbolic metric in $\mathbb{H}_{+}$(resp. in $\mathbb{H}_{-}$) is equivalent with the Euclidean metric in $\mathbb{H}_{+}$(resp. in $\mathbb{H}_{-}$), that is they generate the same topology.

We may extend the hyperbolic topology to the whole space. Indeed, if $U \subset \mathbb{H}$ and the set $U \cap\left\{x \in \mathbb{H} \mid x_{3}=0\right\}$ is non-empty then we call the set $U$ open if it is open with respect to usual Euclidean topology. The inner product $\langle x, y\rangle$ in $\mathbb{H}$ is defined by

$$
\langle x, y\rangle=\sum_{i=0}^{3} x_{i} y_{i}
$$

similarly as in the Euclidean space $\mathbb{R}^{4}$.

The elements

$$
x=x_{0}+x_{1} e_{1}+x_{2} e_{2}
$$

are called reduced quaternions if $x_{0}, x_{1}$ and $x_{2}$ are real numbers. The set of reduced quaternions is identified with $\mathbb{R}^{3}$.

We recall that the prime involution in $\mathbb{H}$ is the mapping $x \rightarrow x^{\prime}$ defined by

$$
x^{\prime}=x_{0}-x_{1} e_{1}-x_{2} e_{2}+x_{3} e_{3} .
$$

Similarly, the reversion in $\mathbb{H}$ is the mapping $x \rightarrow x^{*}$ defined by 


$$
x^{*}=x_{0}+x_{1} e_{1}+x_{2} e_{2}-x_{3} e_{3} .
$$

The conjugation in $\mathbb{H}$ is the mapping $x \rightarrow \bar{x}$ defined by $\bar{x}=\left(x^{\prime}\right)^{*}=\left(x^{*}\right)^{\prime}$, that is

$$
\bar{x}=x_{0}-x_{1} e_{1}-x_{2} e_{2}-x_{3} e_{3} .
$$

These involutions satisfy the following product rules

$$
\begin{aligned}
& (x y)^{\prime}=x^{\prime} y^{\prime}, \\
& (x y)^{*}=y^{*} x^{*}
\end{aligned}
$$

and

$$
\overline{x y}=\bar{y} \bar{x}
$$

for all $x, y \in \mathbb{H}$.

The prime involution may be characterized also as

$$
x e_{3}=e_{3} x^{\prime}
$$

for all quaternions $x$.

The real part of a quaternion $x$ is defined by

$$
\operatorname{Re} x=x_{0}
$$

and the vector part by

$$
\operatorname{Vec} x=x_{1} e_{1}+x_{2} e_{2}+x_{3} e_{3} .
$$

We recall the product rule

$$
x y=-\langle x, y\rangle+x \times y
$$

if $\operatorname{Re} x=\operatorname{Re} y=0$, where $\times$ is the usual cross product in $\mathbb{R}^{3}$.

We define the mappings $S: \mathbb{H} \rightarrow \mathbb{R}^{3}$ and $T: \mathbb{H} \rightarrow \mathbb{R}$ by

$$
S a=a_{0}+a_{1} e_{1}+a_{2} e_{2}
$$

and

$$
T a=a_{3}
$$

for $a=a_{0}+a_{1} e_{1}+a_{2} e_{2}+a_{3} e_{3} \in \mathbb{H}$. Using the reversion, we compute the formulas

$$
\begin{aligned}
& S a=\frac{1}{2}\left(a+a^{*}\right), \\
& T a=-\frac{1}{2}\left(a-a^{*}\right) e_{3} .
\end{aligned}
$$

We recall the identities 


$$
a b+b a=2 a \operatorname{Re} b+2 b \operatorname{Re} a-2\langle a, b\rangle
$$

and

$$
\frac{1}{2}(a \bar{b} c+c \bar{b} a)=\langle b, c\rangle a-[a, b, c]
$$

valid for all quaternions $a, b$ and $c$. The term $[a, b, c]$ is called a triple product and is defined by

$$
[a, b, c]=\langle a, c\rangle b-\langle a, b\rangle c .
$$

If $a, b$ and $c$ are quaternions with $\operatorname{Re} a=\operatorname{Re} b=\operatorname{Re} c=0$, then (cf. [14])

$$
[a, b, c]=a \times(b \times c) .
$$

\section{Hyperregular functions}

We use the following hyperbolic modifications $H_{k}^{l}$ and $H_{k}^{r}$ of the Cauchy-Riemann operators

$$
\begin{array}{ll}
H_{k}^{l} f(x)=D_{l} f(x)+k \frac{f_{3}}{x_{3}}, & \bar{H}_{k}^{l} f(x)=\bar{D}_{l} f(x)-k \frac{f_{3}}{x_{3}}, \\
H_{k}^{r} f(x)=D_{r} f(x)+k \frac{f_{3}}{x_{3}}, & \bar{H}_{k}^{r} f(x)=\bar{D}_{r} f(x)-k \frac{f_{3}}{x_{3}},
\end{array}
$$

where the parameter $k \in \mathbb{R}$ and the generalized Cauchy-Riemann operators are defined by

$$
\begin{array}{rlrl}
D_{l} f & =\sum_{i=0}^{3} e_{i} \frac{\partial f}{\partial x_{i}}, & \bar{D}_{l} f=\sum_{i=0}^{3} \overline{e_{i}} \frac{\partial f}{\partial x_{i}} \\
D_{r} f=\sum_{i=0}^{3} \frac{\partial f}{\partial x_{i}} e_{i}, & \bar{D}_{r} f=\sum_{i=0}^{3} \frac{\partial f}{\partial x_{i}} \overline{e_{i}} .
\end{array}
$$

We also abbreviate $D_{l} f$ by $D f$ and $H_{k}^{l}$ by $H_{k}$.

Definition 1. Let $\Omega \subset \mathbb{H}$ be open. A function $f: \Omega \rightarrow \mathbb{H}$ is called $k$-hyperregular, if $f \in \mathscr{C}^{1}(\Omega)$ and

$$
H_{k}^{l} f(x)=H_{k}^{r} f(x)=0 .
$$

for any $x \in \Omega \backslash\left\{x_{3}=0\right\}$.

We may simply compute the components of the operators $H_{k}^{l}$ and $H_{k}^{r}$ as follows.

Lemma 1. Let $\Omega \subset \mathbb{H}$ be open. If a function $f: \Omega \rightarrow \mathbb{H}$ is differentiable then the coordinate functions of $H_{k}^{l}$ and $H_{k}^{r}$ are given by 


$$
\begin{array}{cc}
\left(H_{k}^{l} f\right)_{0}=\frac{\partial f_{0}}{\partial x_{0}}-\frac{\partial f_{1}}{\partial x_{1}}-\frac{\partial f_{2}}{\partial x_{2}}-\frac{\partial f_{3}}{\partial x_{3}}+k \frac{f_{3}}{x_{3}}, & \left(H_{k}^{r} f\right)_{0}=\left(H_{k}^{l} f\right)_{0}, \\
\left(H_{k}^{l} f\right)_{1}=\frac{\partial f_{0}}{\partial x_{1}}+\frac{\partial f_{1}}{\partial x_{0}}-\frac{\partial f_{2}}{\partial x_{3}}+\frac{\partial f_{3}}{\partial x_{2}}, & \left(H_{k}^{r} f\right)_{1}=\frac{\partial f_{0}}{\partial x_{1}}+\frac{\partial f_{1}}{\partial x_{0}}+\frac{\partial f_{2}}{\partial x_{3}}-\frac{\partial f_{3}}{\partial x_{2}}, \\
\left(H_{k}^{l} f\right)_{2}=\frac{\partial f_{0}}{\partial x_{2}}+\frac{\partial f_{2}}{\partial x_{0}}+\frac{\partial f_{1}}{\partial x_{3}}-\frac{\partial f_{3}}{\partial x_{1}}, & \left(H_{k}^{r} f\right)_{2}=\frac{\partial f_{0}}{\partial x_{2}}+\frac{\partial f_{2}}{\partial x_{0}}-\frac{\partial f_{1}}{\partial x_{3}}+\frac{\partial f_{3}}{\partial x_{1}}, \\
\left(H_{k}^{l} f\right)_{3}=\frac{\partial f_{0}}{\partial x_{3}}+\frac{\partial f_{3}}{\partial x_{0}}-\frac{\partial f_{1}}{\partial x_{2}}+\frac{\partial f_{2}}{\partial x_{1}}, & \left(H_{k}^{r} f\right)_{3}=\frac{\partial f_{0}}{\partial x_{3}}+\frac{\partial f_{3}}{\partial x_{0}}+\frac{\partial f_{1}}{\partial x_{2}}-\frac{\partial f_{2}}{\partial x_{1}},
\end{array}
$$

where $(\cdot)_{j}$ denotes the real coefficient of the element $e_{j}$ for each $j=0,1,2,3$.

We obtain immediately the following result.

Proposition 2. Let $\Omega \subset \mathbb{H}$ be open and a function $f: \Omega \rightarrow \mathbb{H}$ continuously differentiable. A function $f$ is $k$-hyperregular in $\Omega$ if and only if

$$
\begin{gathered}
\frac{\partial f_{0}}{\partial x_{0}}-\frac{\partial f_{1}}{\partial x_{1}}-\frac{\partial f_{2}}{\partial x_{2}}-\frac{\partial f_{3}}{\partial x_{3}}+k \frac{f_{3}}{x_{3}}=0, \text { if } x_{3} \neq 0, \\
\frac{\partial f_{0}}{\partial x_{i}}=-\frac{\partial f_{i}}{\partial x_{0}} \text { for all } i=1,2,3, \\
\frac{\partial f_{i}}{\partial x_{j}}=\frac{\partial f_{j}}{\partial x_{i}} \text { for all } i, j=1,2,3 .
\end{gathered}
$$

Our operators are connected to the hyperbolic metric via the hyperbolic Laplace operator as follows.

Proposition 3. Let $f: \Omega \rightarrow \mathbb{H}$ be twice continuously differentiable. Then

$$
\begin{aligned}
H_{k}^{l} \bar{H}_{k}^{l} f= & \Delta f-\frac{k}{x_{3}} \frac{\partial f}{\partial x_{3}}+\frac{k f_{3}}{x_{3}^{2}} e_{3}+\frac{k}{x_{3}}\left(\frac{\partial f_{1}}{\partial x_{2}}-\frac{\partial f_{2}}{\partial x_{1}}\right) \\
& +\frac{k}{x_{3}}\left(\frac{\partial f_{1}}{\partial x_{3}}-\frac{\partial f_{3}}{\partial x_{1}}\right) e_{1}+\frac{k}{x_{3}}\left(\frac{\partial f_{2}}{\partial x_{3}}-\frac{\partial f_{3}}{\partial x_{2}}\right) e_{2} \\
= & \bar{H}_{k}^{l} H_{k}^{l} f
\end{aligned}
$$

and

$$
\begin{aligned}
H_{k}^{r} \bar{H}_{k}^{r} f= & \Delta f-\frac{k}{x_{3}} \frac{\partial f}{\partial x_{3}}+\frac{k f_{3} e_{3}}{x_{3}^{2}}+\frac{k}{x_{3}}\left(\frac{\partial f_{2}}{\partial x_{1}}-\frac{\partial f_{1}}{\partial x_{2}}\right) \\
& +\frac{k}{x_{3}}\left(\frac{\partial f_{1}}{\partial x_{3}}-\frac{\partial f_{3}}{\partial x_{1}}\right) e_{1}+\frac{k}{x_{3}}\left(\frac{\partial f_{2}}{\partial x_{3}}-\frac{\partial f_{3}}{\partial x_{2}}\right) e_{2} \\
= & \bar{H}_{k}^{r} H_{k}^{r} f .
\end{aligned}
$$

Proof. We just compute

$$
\begin{aligned}
D_{l} \overline{H_{k}^{l}} f & =D_{l} \bar{D}_{l} f-k \frac{D f_{3}}{x_{3}}+\frac{k f_{3} e_{3}}{x_{3}^{2}} \\
& =\Delta f-k \frac{\frac{\partial f_{3}}{\partial x_{0}}+\frac{\partial f_{3}}{\partial x_{1}} e_{1}+\frac{\partial f_{3}}{\partial x_{2}} e_{2}+\frac{\partial f_{3}}{\partial x_{3}} e_{3}}{x_{3}}+\frac{k f_{3} e_{3}}{x_{3}^{2}}
\end{aligned}
$$

and 


$$
\left(\bar{H}_{k}^{l} f\right)_{3}=\left(\bar{D}_{l} f\right)_{3}=-\frac{\partial f_{0}}{\partial x_{3}}+\frac{\partial f_{1}}{\partial x_{2}}-\frac{\partial f_{2}}{\partial x_{1}}+\frac{\partial f_{3}}{\partial x_{0}} .
$$

Hence we obtain

$$
\begin{aligned}
H_{k}^{l} \bar{H}_{k}^{l} f= & \Delta f-\frac{k}{x_{3}} \frac{\partial f}{\partial x_{3}}+\frac{k f_{3}}{x_{3}^{2}} e_{3}+\frac{k}{x_{3}}\left(\frac{\partial f_{1}}{\partial x_{2}}-\frac{\partial f_{2}}{\partial x_{1}}\right) \\
& +\frac{k}{x_{3}}\left(\frac{\partial f_{1}}{\partial x_{3}}-\frac{\partial f_{3}}{\partial x_{1}}\right) e_{1}+\frac{k}{x_{3}}\left(\frac{\partial f_{2}}{\partial x_{3}}-\frac{\partial f_{3}}{\partial x_{2}}\right) e_{2} .
\end{aligned}
$$

Similarly, we compute

$$
\begin{aligned}
D_{r} \overline{H_{k}^{r}} f & =D_{r} \bar{D}_{r} f-k \frac{D_{r} f_{3}}{x_{3}}+\frac{k f_{3} e_{3}}{x_{3}^{2}} \\
& =\Delta f-k \frac{\frac{\partial f_{3}}{\partial x_{0}}+\frac{\partial f_{3}}{\partial x_{1}} e_{1}+\frac{\partial f_{3}}{\partial x_{2}} e_{2}+\frac{\partial f_{3}}{\partial x_{3}} e_{3}}{x_{3}}+\frac{k f_{3} e_{3}}{x_{3}^{2}}
\end{aligned}
$$

and

$$
\left(\bar{H}_{k}^{r} f\right)_{3}=\left(\bar{D}_{r} f\right)_{3}=-\frac{\partial f_{0}}{\partial x_{3}}-\frac{\partial f_{1}}{\partial x_{2}}+\frac{\partial f_{2}}{\partial x_{1}}+\frac{\partial f_{3}}{\partial x_{0}} .
$$

Hence we have

$$
\begin{aligned}
H_{k}^{r} \bar{H}_{k}^{r} f= & \Delta f-\frac{k}{x_{3}} \frac{\partial f}{\partial x_{3}}+\frac{k f_{3} e_{3}}{x_{3}^{2}}+\frac{k}{x_{3}}\left(\frac{\partial f_{2}}{\partial x_{1}}-\frac{\partial f_{1}}{\partial x_{2}}\right) \\
& +\frac{k}{x_{3}}\left(\frac{\partial f_{1}}{\partial x_{3}}-\frac{\partial f_{3}}{\partial x_{1}}\right) e_{1}+\frac{k}{x_{3}}\left(\frac{\partial f_{2}}{\partial x_{3}}-\frac{\partial f_{3}}{\partial x_{2}}\right) e_{2} .
\end{aligned}
$$

Moreover, we easily deduce that $\bar{H}_{k}^{l} H_{k}^{l} f=H_{k}^{l} \bar{H}_{k}^{l} f$ and $\bar{H}_{k}^{r} H_{k}^{r} f=H_{k}^{r} \bar{H}_{k}^{r} f$.

We immediately obtain two corollaries.

Corollary 2. If $f: \Omega \rightarrow \mathbb{H}$ is twice continuously differentiable and $k \neq 0$ then

$$
H_{k}^{l} \bar{H}_{k}^{l} f=H_{k}^{r} \bar{H}_{k}^{r} f=\Delta f-\frac{k}{x_{3}} \frac{\partial f}{\partial x_{3}}+\frac{k f_{3} e_{3}}{x_{3}^{2}}
$$

if and only if $\frac{\partial f_{i}}{\partial x_{j}}=\frac{\partial f_{j}}{\partial x_{i}}$ for all $i, j=1,2,3$.

Corollary 3. If $f: \Omega \rightarrow \mathbb{R}$ is real vakued and twice continuously differentiable then

$$
x_{3}^{k} H_{k}^{l} \bar{H}_{k}^{l} f=x_{3}^{k} H_{k}^{r} \bar{H}_{k}^{r} f=\Delta_{k} f
$$

where the operator

$$
\Delta_{k}=x_{3}^{k}\left(\Delta-\frac{k}{x_{3}} \frac{\partial}{\partial x_{3}}\right)
$$

is the Laplace-Beltrami operator (see [19]) with respect to the Riemannian metric 


$$
d s_{k}^{2}=\frac{d x_{0}^{2}+d x_{1}^{2}+d x_{2}^{2}+d x_{3}^{2}}{x_{3}^{k}} .
$$

Differentiating the first equation of Proposition 2 with respect to $x_{i}$ and applying the rest of the equations of Proposition 2 we obtain the following result.

Proposition 4. Let $\Omega \subset \mathbb{H}$ be open and a function $f: \Omega \rightarrow \mathbb{H}$ twice continuously differentiable. If $f$ is $k$-hyperregular then

$$
x_{3}^{k} H_{k}^{l} \bar{H}_{k}^{l} f=x_{3}^{k} H_{k}^{r} \bar{H}_{k}^{r} f=\Delta_{k} f+x_{3}^{k-2} k f_{3} e_{3}=0 .
$$

The previous results motivate the following definition.

Definition 2. Let $\Omega \subset \mathbb{H}$ be open. A twice continuously differentiable function $f$ : $\Omega \rightarrow \mathbb{H}$ is called $k$-hyperbolic, if

$$
\Delta f-\frac{k}{x_{3}} \frac{\partial f}{\partial x_{3}}+\frac{k f_{3} e_{3}}{x_{3}^{2}}=0 .
$$

There exists a characterization of $k$-hyperregular functions in terms of $k$-hyperbolic functions.

Theorem 1. Let $\Omega \subset \mathbb{H}$ be open. A twice continuously differentiable hyperbolic harmonic function $f: \Omega \rightarrow \mathbb{H}$ is $k$-hyperregular if and only if the functions $f$ and $x f+f x$ are $k$-hyperbolic and $H_{k}^{l} f=H_{k}^{r} f$.

Proof. In order to abbreviate notations, we denote $g=x f+f x$. Using the standard formulas $\Delta(x f)=x \Delta f+2 D_{l} f$ and $\Delta(f x)=(\Delta f) x+2 D_{r} f$ we obtain by virtue of Proposition 4, that

$$
\begin{aligned}
x_{3}^{2} \Delta g-k x_{3} \frac{\partial g}{\partial x_{3}}+k g_{3} e_{3}= & x_{3}^{2} x H_{k}^{l} \bar{H}_{k}^{l} f+x_{3}^{2}\left(H_{k}^{l} \bar{H}_{k}^{l} f\right) x+2 x_{3}^{2} H_{k}^{l} f+2 x_{3}^{2} H_{k}^{r} f \\
& -4 k x_{3} f_{3}-k x_{3}\left(e_{3} f^{\prime}+f e_{3}\right)+2 k\left(x_{0} f_{3}+x_{3} f_{0}\right) e_{3}+ \\
& -2 k f_{3}\left(x_{0} e_{3}-x_{3}\right) \\
= & x_{3}^{2} x H_{k}^{l} \bar{H}_{k}^{l} f+x_{3}^{2}\left(H_{k}^{l} \bar{H}_{k}^{l} f\right) x \\
& +2 x_{3}^{2} H_{k}^{l} f+2 x_{3}^{2} H_{k}^{r} f .
\end{aligned}
$$

If $f$ is $k$-hyperregular then

$$
x_{3}^{2} H_{k}^{l} \bar{H}_{k}^{l} f=x_{3}^{2} \Delta f-k x_{3} \frac{\partial f}{\partial x_{3}}+k f_{3} e_{3}=0
$$

and $H_{k}^{l} f=H_{k}^{r} f=0$ which implies that $g$ is $k$-hyperbolic. Conversely, if $g$ and $f$ are $k$-hyperbolic and $H_{k}^{l} f=H_{k}^{r} f$ then

$$
H_{k}^{l} f+H_{k}^{r} f=0 .
$$


Hence $f$ is $k$-hyperregular.

Real valued $k$-hyperbolic functions are especially important, since they produce $k$-hyperregular functions.

Theorem 2. Let $\Omega$ be an open subset of $\mathbb{H}$. If $h$ is real valued $k$-hyperbolic on $\Omega$ then the function $f=\bar{D} h$ is $k$-hyperregular on $\Omega$. Conversely, if $f$ is $k$-hyperregular on $\Omega$, there exists locally a real valued $k$-hyperbolic function $h$ satisfying $f=\bar{D} h$.

Proof. Let $h$ be real $k$ - hyperbolic on $\Omega$ and denote $f=\bar{D} h$. Applying Proposition 3 we obtain

$$
H_{k}^{l} f=H_{k}^{l} \bar{H}_{k}^{l} h=\Delta h-\frac{k}{x_{3}} \frac{\partial h}{\partial x_{3}}=0=H_{k}^{r} \bar{H}_{k}^{r} h=H_{k}^{r} f .
$$

Hence $f$ is $k$-hyperregular. The converse statement is verified similarly as in [7].

We use the following transformation property proved in [5].

Lemma 2. Let $\Omega$ be an open set contained in $\mathbb{H}_{+}$or in $\mathbb{H}_{-}$. A function $f: \Omega \rightarrow$ $\mathbb{R}$ is $k$-hyperbolic harmonic if and only if the function $g(x)=x_{3}^{\frac{2-k}{2}} f(x)$ satisfies the equation

$$
\Delta_{2} S g+\frac{1}{4}\left(9-(k+1)^{2}\right) S g=0
$$

\section{Cauchy type integral formulas}

We first recall the quaternionic version of the Stokes theorem verified for example in [14] as follows. If $\Omega$ is an open subset of $\mathbb{H}, K$ a 3-chain satisfying $\bar{K} \subset \Omega$ and $f, g \in \mathscr{C}^{1}(\Omega, \mathbb{H})$, then

$$
\int_{\partial K} g v f d \sigma=\int_{K}\left(D_{r} g f+g D_{l} f\right) d m
$$

where $v=v_{0}+v_{1} e_{1}+v_{2} e_{2}+v_{3} e_{3}$ is the outer normal, $d \sigma$ the surface element and $d m$ is the usual Lebesgue volume element in $\mathbb{R}^{4}$ identified with $\mathbb{H}$ as a vector space.

The $T$-part and $S$-part play a strong role in our operator $H_{k}$. We have therefore two versions of the Stokes theorem. The first version deals with $T$-part and the second one with $S$-part.

Theorem 3. Let $\Omega$ be an open subset of $\mathbb{H} \backslash\left\{x_{3}=0\right\}$ and $K$ a 3-chain satisfying $\bar{K} \subset \Omega$. If $f, g \in \mathscr{C}^{1}(\Omega, \mathbb{H})$, then

$$
\int_{\partial K} g v f d \sigma=\int_{K}\left(\left(H_{-k}^{r} g\right) f+g H_{k}^{l} f+\frac{k}{x_{3}}\left(\left(g_{3}\right) S f-S g f_{3}\right)\right) d m
$$

and therefore 


$$
T\left(\int_{\partial K} g v f d \sigma\right)=\int_{K} T\left(\left(H_{-k}^{r} g\right) f+g H_{k}^{l} f\right) d m
$$

where $v=v_{0}+v_{1} e_{1}+v_{2} e_{2}+v_{3} e_{3}$ is the outer normal, $d \sigma$ the surface element and $d m$ is the usual Lebesgue volume element in $\mathbb{R}^{4}$.

Proof. Since $D_{r} g=H_{-k}^{r} g+k \frac{g_{3}}{x_{3}}$ and $D_{l} f=H_{k}^{l} f-k \frac{f_{3}}{x_{3}}$ we deduce using (10) that

$$
\begin{aligned}
\int_{\partial K}(g d \sigma f) & =\int_{K}\left(\left(H_{-k}^{r} g\right) f+g H_{k}^{l} f+\frac{k}{x_{3}}\left(\left(g_{3}\right) f-g f_{3}\right)\right) d m \\
& =\int_{K}\left(\left(H_{-k}^{r} g\right) f+g H_{k}^{l} f+\frac{k}{x_{3}}\left(\left(g_{3}\right) S f-S g f_{3}\right)\right) d m
\end{aligned}
$$

completing the proof.

We may also prove

Theorem 4. Let $\Omega$ be an open subset of $\mathbb{H}^{4} \backslash\left\{x_{3}=0\right\}$ and $K$ a 3 -chain satisfying $\bar{K} \subset \Omega$. If $f, g \in \mathscr{C}^{1}(\Omega, \mathbb{H})$, then

$$
\int_{\partial K} f v g d \sigma=\int_{K}\left(\left(H_{k}^{r} f\right) g+f H_{-k}^{l} g+\frac{k}{x_{3}}\left(\left(g_{3}\right) S f-S g f_{3}\right)\right) d m
$$

and therefore

$$
T\left(\int_{\partial K} f v g d \sigma\right)=\int_{K} T\left(\left(H_{k}^{r} f\right) g+f H_{-k}^{l} g\right) d m
$$

where $v=v_{0}+v_{1} e_{1}+v_{2} e_{2}+v_{3} e_{3}$ is the outer normal, $d \sigma$ the surface element and $d m$ is the usual Lebesgue volume element in $\mathbb{R}^{4}$.

Proof. Since $D_{l} g=H_{-k}^{l} g+k \frac{g_{3}}{x_{3}}$ and $D_{r} f=H_{k}^{r} f-k \frac{f_{3}}{x_{3}}$ we deduce using (10) that

$$
\begin{aligned}
\int_{\partial K}(g v f) d \sigma & =\int_{K}\left(\left(H^{r} f\right) g+f H_{-k}^{l} g+\frac{k}{x_{3}}\left(f g_{3}-f_{3} g\right)\right) d m \\
& =\int_{K}\left(\left(H^{r} f\right) f+g H_{-k}^{l} g+\frac{k}{x_{3}}\left(\left(g_{3}\right) S f-S g f_{3}\right)\right) d m
\end{aligned}
$$

completing the proof.

Combining previous results we conclude the result

Theorem 5. Let $\Omega$ be an open subset of $\mathbb{R}^{4} \backslash\left\{x_{3}=0\right\}$ and $K$ a 3 -chain satisfying $\bar{K} \subset \Omega$. If $f, g \in \mathscr{C}^{1}(\Omega, \mathbb{H})$, then

$$
\int_{\partial K} T(g \vee f+f v g) d \sigma=\int_{K} T\left(H_{-k}^{r} g f+g H_{k}^{l} f+H_{k}^{r} f g+f H_{-k}^{l} g\right) d m
$$

where $v=v_{0}+v_{1} e_{1}+v_{2} e_{2}+v_{3} e_{3}$ is the outer normal, $d \sigma$ the surface element and $d m$ is the usual Lebesgue volume element in $\mathbb{R}^{4}$. 
Theorem 6. Let $\Omega$ be an open subset of $\mathbb{R}^{4} \backslash\left\{x_{3}=0\right\}$ and $K$ a 3-chain satisfying $\bar{K} \subset \Omega$. If $f, g \in \mathscr{C}^{1}(\Omega, \mathbb{H})$, then

$$
\int_{\partial K} S(g v f+f v g) \frac{d \sigma}{x_{3}^{k}}=\int_{K} S\left(H_{k}^{r} g f+g H_{k}^{l} f+H_{k}^{r} f g+f H_{k}^{l} g\right) \frac{d m}{x_{3}^{k}},
$$

where $v=v_{0}+v_{1} e_{1}+v_{2} e_{2}+v_{3} e_{3}$ is the outer normal, $d \sigma$ the surface element and $d m$ is the usual Lebesgue volume element in $\mathbb{R}^{4}$.

Proof. Applying (10), we deduce

$$
\int_{\partial K} g v f \frac{d \sigma}{x_{3}^{k}}=\int_{K}\left(D_{r} g f+g D_{l} f-k \frac{g e_{3} f}{x_{3}}\right) \frac{d m}{x_{3}^{k}} .
$$

Since $H_{k}^{r} g=D_{r} g+\frac{k g_{3}}{x_{3}}$ and $H_{k}^{l} f=D_{l} g+\frac{k f_{3}}{x_{3}}$, we infer

$$
\int_{\partial K} g v f \frac{d \sigma}{x_{3}^{k}}=\int_{K}\left(H_{k}^{r} g f+g H_{k}^{l} f-k \frac{g_{3} f+g f_{3}+g e_{3} f}{x_{3}}\right) \frac{d m}{x_{3}^{k}} .
$$

Using the formula $g e_{3} f=g e_{3} S f-g f_{3}$, we obtain

$$
\begin{aligned}
\int_{\partial K} g v f \frac{d \sigma}{x_{3}^{k}} & =\int_{K}\left(H_{k}^{r} g f+g H_{k}^{l} f-k \frac{g_{3} f+g e_{3} S f}{x_{3}}\right) \frac{d m}{x_{3}^{k}} \\
& =\int_{K}\left(H_{k}^{r} g f+g H_{k}^{l} f-k \frac{g_{3} f_{3} e_{3}+S g e_{3} S f}{x_{3}}\right) \frac{d m}{x_{3}^{k}} .
\end{aligned}
$$

If we compute the coordinates of $S g e_{3} S f$, we have

$$
\begin{aligned}
\int_{\partial K} g v f \frac{d \sigma}{x_{3}^{k}}= & \int_{K}\left(H_{k}^{r} g f+g H_{k}^{l} f-k \frac{g_{0} f_{0}+g_{1} f_{1}+g_{2} f_{2}+g_{3} f_{3}}{x_{3}} e_{3}\right) \frac{d m}{x_{3}^{k}} \\
& -\int_{K} k \frac{g_{1} f_{2}-g_{2} f_{1}+\left(g_{2} f_{0}-g_{0} f_{2}\right) e_{1}+\left(g_{0} f_{1}-g_{1} f_{0}\right) e_{2}}{x_{3}^{k+1}} d m .
\end{aligned}
$$

If we interchange the roles of $f$ and $g$, we infer

$$
\begin{aligned}
\int_{\partial K} f v g \frac{d \sigma}{x_{3}^{k}}= & \int_{K}\left(H_{k}^{r} f g+f H_{k}^{l} g-k \frac{g_{0} f_{0}+g_{1} f_{1}+g_{1} f_{1}+g_{3} f_{3}}{x_{3}} e_{3}\right) \frac{d m}{x_{3}^{k}} \\
& -\int_{K} k \frac{f_{1} g_{2}-f_{2} g_{1}+\left(f_{2} g_{0}-f_{0} g_{2}\right) e_{1}+\left(f_{0} g_{1}-f_{1} g_{0}\right) e_{2}}{x_{3}^{k+1}} d m
\end{aligned}
$$

Hence 


$$
\begin{aligned}
\int_{\partial K}(g \vee f+f v g) \frac{d \sigma}{x_{3}^{k}}= & \int_{K}\left(H_{k}^{r} g f+g H_{k}^{l} f+H_{k}^{r} f g+f H_{k}^{l} g\right) \frac{d m}{x_{3}^{k}} \\
& -2 k e_{3} \int_{K} \frac{g_{0} f_{0}+g_{1} f_{1}+g_{1} f_{1}+g_{3} f_{3}}{x_{3}} \frac{d m}{x_{3}^{k}}
\end{aligned}
$$

and therefore

$$
\int_{\partial K} S(g v f+f v g) \frac{d \sigma}{x_{3}^{k}}=\int_{K} S\left(H_{k}^{r} g f+g H_{k}^{l} f+H_{k}^{r} f g+f H_{k}^{l} g\right) \frac{d m}{x_{3}^{k}} .
$$

The hyperbolic Laplace operator of functions depending on $\lambda$ is computed in [5] as follows.

Lemma 3. Let $x$ and $y$ be poins in the upper half space. If $f$ is twice continuously differentiable depending only on $\lambda=\lambda(x, y)$, then

$$
\Delta_{h} f(x)=\left(\lambda^{2}-1\right) \frac{\partial^{2} f}{\partial \lambda^{2}}+4 \lambda \frac{\partial f}{\partial \lambda} .
$$

We recall the definition of the associated Legendre function of the second kind

$$
Q_{v}^{\mu}(\lambda)=C\left(\lambda^{2}-1\right)^{\frac{\mu}{2}} \lambda^{-v-\mu-1}{ }_{2} F_{1}\left(\frac{v+\mu+2}{2}, \frac{\mu+v+1}{2} ; \frac{2 v+3}{2} ; \frac{1}{\lambda^{2}}\right)
$$

where

$$
C=-\frac{\sqrt{\pi} \Gamma(v+\mu+1)}{2^{v+1} \Gamma\left(v+\frac{3}{2}\right)} .
$$

and the hypergeometric function is defined by

$$
{ }_{2} F_{1}(a, b ; c ; x)=\sum_{m=0}^{\infty} \frac{(a)_{m}(b)_{m}}{(c)_{m}} \frac{x^{m}}{m !},
$$

converging in the usual sense at least for $x$ satisfying $|x|<1$. Associated Legendre functions satisfies the differential equation (see [20])

$$
\left(\lambda^{2}-1\right) u^{\prime \prime}(\lambda)+2 \lambda u^{\prime}(\lambda)-\left(v(v+1)-\frac{\mu^{2}}{1-\lambda^{2}}\right) u(\lambda)=0 .
$$

We are looking for solutions of the equation

$$
\Delta_{h} f(\lambda)+\gamma f(\lambda)=0
$$

in the form

$$
f(\lambda)=\left(\lambda^{2}-1\right)^{\alpha} g(\lambda) .
$$

We just compute that 


$$
\left(\lambda^{2}-1\right) g^{\prime \prime}(\lambda)+(4 \alpha+4) \lambda g^{\prime}(\lambda)+\left(4 \alpha^{2}+6 \alpha+\gamma+\frac{2 \alpha(2+2 \alpha)}{\lambda^{2}-1}\right) g(\lambda)=0 .
$$

In order to compute the solutions using Legendre functions, we compare this equation with (11) and first we set $4 \alpha+4=2$ and therefore $\alpha=-\frac{1}{2}$. Then we have the equation

$$
\left(\lambda^{2}-1\right) g^{\prime \prime}(\lambda)+2 \lambda g^{\prime}(\lambda)+\left(-2+\gamma-\frac{1}{1-\lambda^{2}}\right) g(\lambda)=0
$$

and again comparing with (11), we obtain equations

$$
\begin{aligned}
v(v+1) & =2-\gamma, \\
\mu^{2} & =\frac{(n-1)^{2}}{4} .
\end{aligned}
$$

Hence $\mu= \pm 1$ and $v=\frac{\sqrt{9-4 \gamma}-1}{2}$. Setting $-\gamma=\frac{1}{4}\left((k+1)^{2}-9\right)$, we obtain

$$
v=\frac{ \pm|k+1|-1}{2} \text {. }
$$

Consequently, we found a solution $\left(\lambda^{2}-1\right)^{-\frac{1}{2}} Q_{\frac{|k+1|-1}{2}}^{1}(\lambda)$. Note that $Q_{\frac{|k+1|-1}{2}}^{1}(\lambda)$ is well defined since $\lambda>1$ and $\frac{|k+1|-1}{2}>-1$.

Denote $v=\frac{|k+1|-1}{2}$. Applying [20, S.2.9-4.] and the definition of $Q_{v}^{1}(\lambda)$, we obtain

$$
\begin{aligned}
Q_{v}^{1}(\lambda) & =-\frac{v+1}{2^{v+1}} \frac{\int_{0}^{\pi}(\lambda+\cos \alpha)^{-v} \sin ^{2 v+1} \alpha d \alpha}{\left(\lambda^{2}-1\right)^{\frac{1}{2}}} \\
& =-\frac{\sqrt{\pi} \Gamma(v+2) \lambda^{-v}{ }_{2} F_{1}\left(\frac{v}{2}, \frac{v+1}{2} ; \frac{2 v+3}{2} ; \frac{1}{\lambda^{2}}\right)}{2^{v+1} \Gamma\left(v+\frac{3}{2}\right)\left(\lambda^{2}-1\right)} .
\end{aligned}
$$
is

We recall that the volume measure of the Riemannian metric $d s_{k}$ defined in (8)

$$
d m_{k}=y_{3}^{-2 k} d m
$$

where $d m$ is the usual Lebesgue measure. Its surface element is defined by $d \sigma_{(k)}=$ $y_{3}^{-\frac{3 k}{2}} d \sigma$. The outer normal in $\partial B_{h}\left(x, R_{h}\right)$ is denoted by $n_{e}$ and the outer normal derivative is defined by $\frac{\partial u}{\partial n^{k}}=y_{3}^{\frac{k}{2}} \frac{\partial u}{\partial n_{e}}$.

We prove that the function

$$
F_{k}(x, y)=-\frac{x_{3}^{\frac{k-2}{2}} y_{3}^{\frac{k-2}{2}} Q_{v}^{1}\left(\cosh d_{h}(x, y)\right)}{\omega_{3} \sinh d_{h}(x, y)}
$$


is the fundamental $k$-hyperbolic harmonic function at the point $x$ (symmetrically $y$ ), that is $-\Delta_{k} F_{k}=\delta_{x}$ in the distributional sense with respect to the volume measure of the Riemannian metric $d s_{k}$ and $\omega_{3}=2 \pi^{2}$ is the Euclidean surface area of the unit ball in $\mathbb{H}$. We also remind that the fundamental $k$-harmonic function is unique up to the $k$-hyperbolic harmonic function.

We first verify the following crucial result.

Lemma 4. Let $x$ be a point in the upper half space and denote $v=\frac{|k+1|-1}{2}$. The function

$$
\begin{aligned}
g_{k}\left(d_{h}(x, y)\right) & =\frac{v+1}{2^{v+1}} \int_{0}^{\pi}\left(\cosh d_{h}(x, y)+\cos \alpha\right)^{-v} \sin ^{2 v+1} \alpha d \alpha \\
& =\frac{\sqrt{\pi} \Gamma(v+2) \lambda^{-v}{ }_{2} F_{1}\left(\frac{v}{2}, \frac{v+1}{2} ; \frac{2 v+3}{2} ; \frac{1}{\cosh ^{2} d_{h}(x, y)}\right)}{2^{v+1} \Gamma\left(v+\frac{3}{2}\right)}
\end{aligned}
$$

is positive and continuous for any $y \in \mathbb{H}_{+}$and

$$
g_{k}(0)=1
$$

Proof. Applying properties of hypergeometric functions (see for example [2]) and the Gamma function, we infer that

$$
{ }_{2} F_{1}\left(\frac{v}{2}, \frac{v+1}{2} ; \frac{2 v+3}{2} ; 1\right)=\frac{\Gamma\left(v+\frac{3}{2}\right) \Gamma(1)}{\Gamma\left(\frac{v+3}{2}\right) \Gamma\left(\frac{v+2}{2}\right)}=\frac{\Gamma\left(v+\frac{3}{2}\right) 2^{v+1}}{\sqrt{\pi} \Gamma(v+2)} .
$$

Hence $g_{k}(0)=1$.

Next we prove that $F_{k}(x, y)$ is integrable in the hyperbolic ball $B_{h}\left(a, R_{h}\right)$ with respect to the Riemannian volume measure $d m_{k}$.

Lemma 5. The function $F_{k}(x, y)$ is integrable in the hyperbolic ball $B_{h}\left(x, R_{h}\right)$ with respect to the volume measure $d m_{k}$ in the hyperbolic ball $B_{h}\left(x, R_{h}\right)$ and

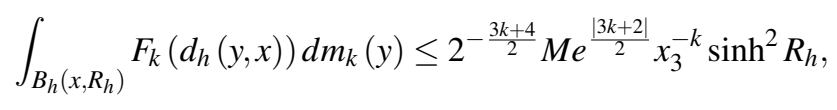

where $M=\max _{y \in \overline{B_{h}\left(x, R_{h}\right)}}\left(g_{k}(y, x)\right) \geq 1$.

Proof. Using Proposition 1 we infer that the hyperbolic ball $B_{h}\left(x, R_{h}\right)$ is an Euchlidean ball with the Euchlidean center $c_{x}\left(R_{h}\right)=x_{0}+x_{1} e_{1}+x_{2} e_{2}+x_{2} \cosh R_{h}$ and the Euchlidean radius $R_{e}=x_{3} \sinh R_{h}$. Hence we deduce

$$
\frac{g_{k}\left(d_{h}(x, y)\right)}{x_{3}^{2} \sinh ^{2} d_{h}(y, x)}=\frac{g_{k}\left(d_{h}(x, y)\right)}{\left\|y-c_{x}\left(R_{h}\right)\right\|^{2}}
$$

and in $B_{h}\left(x, R_{h}\right)$ 


$$
2 x_{3} e^{-R_{h}}=x_{3}\left(\cosh R_{h}-\sinh R_{h}\right) \leq y_{3} \leq x_{3}\left(\cosh R_{h}+\sinh R_{h}\right)=2 x_{3} e^{R_{h}}
$$

for all $y \in B_{h}\left(x, R_{h}\right)$. Since $g_{k}\left(d_{h}(x, y)\right)$ is a continuous function, it attains its maximum in the closure of the ball $B_{h}\left(x, R_{h}\right)$. Since

$$
\begin{aligned}
\int_{B_{h}\left(x, R_{h}\right)} x_{3}^{-2} \sinh ^{-2} d_{h}(y, x) d m(y) & =\int_{B_{e}\left(c_{x}\left(R_{h}\right), x_{3} \sinh R_{h}\right)} \frac{d m(y)}{\left\|y-c_{x}\left(R_{h}\right)\right\|^{2}} \\
& =\int_{0}^{x_{3} \sinh R_{h}} r \int_{\partial B_{h}\left(c_{x}\left(r_{h}\right), 1\right)} d S d r \\
& =\frac{\omega_{3} x_{3}^{2} \sinh ^{2} R_{h}}{2}
\end{aligned}
$$

we conclude

$$
\int_{B_{h}\left(x, R_{h}\right)} F_{k}(y, x) d m_{k}(y) \leq 2^{-\frac{3 k+4}{2}} M e^{\frac{|3 k+2|}{2}} x_{3}^{-k} \sinh ^{2} R_{h} .
$$

We also need the result

Lemma 6. Let $\Omega \subset \mathbb{H}_{+}$be open and $\overline{B_{h}\left(x, R_{h}\right)} \subset \Omega$. Let $u$ be a continuous real valued function in $\Omega$. Then

$$
\lim _{R_{h} \rightarrow 0} \int_{\partial B_{h}\left(x, R_{h}\right)} u \frac{\partial F_{k}(x, y)}{\partial n^{k}} d \sigma_{(k)}(y)=-u(x)
$$

Proof. Applying Proposition 1 we obtain that the outer normal at $y \in \partial B_{h}\left(x, R_{h}\right)$ is

$$
n_{e}=\left(n_{0}, n_{1}, n_{2}, n_{3}\right)=\frac{\left(y_{0}-x_{0}, y_{1}-x_{1}, y_{2}-x_{2}, y_{3}-x_{3} \cosh R_{h}\right)}{x_{3} \sinh R_{h}}
$$

In order to abbreviate the notations, we denote briefly $r_{h}=d_{h}(y, x)$. We compute the outer normal derivative by

$$
\begin{aligned}
\frac{\partial F_{k}(x, y)}{\partial n^{k}}= & y_{3}^{\frac{k}{2}} \frac{\partial F_{k}(x, y)}{\partial n_{e}}=y_{3}^{\frac{k}{2}}\left\langle n_{e}, \operatorname{grad} F_{k}(x, y)\right\rangle \\
= & y_{3}^{k-1} x_{3}^{\frac{k-2}{2}} \frac{\partial \frac{g_{k}\left(r_{h}\right)}{\sinh ^{2} r_{h}}}{\partial r_{h}} \sum_{i=0}^{3} n_{i} \frac{\partial r_{h}}{\partial y_{i}} \\
& +\frac{k-2}{2} y_{3}^{\frac{k-2}{2}} n_{3} F_{k}(x, y) .
\end{aligned}
$$

Since $r_{h}=\operatorname{arcos} \lambda(\mathrm{y}, \mathrm{x})$ we deduce

$$
\frac{\partial r_{h}}{\partial y_{i}}=\frac{\partial \arccos \lambda(y, x)}{\partial y_{i}}=\frac{y_{i}-x_{i}-x_{3}\left(\cosh r_{h}-1\right) \delta_{i 3}}{y_{3} x_{3} \sinh r_{h}}
$$

and therefore the identity 


$$
\sum_{i=0}^{3} n_{i} \frac{\partial r_{h}}{\partial y_{i}}=\frac{1}{y_{3}}
$$

holds. Hence we compute further

$$
\begin{aligned}
\frac{\partial F_{k}(x, y)}{\partial n^{k}}= & \frac{y_{3}^{k-2} x_{3}^{\frac{k-2}{2}}}{\omega_{3} \sinh ^{2} r_{h}} \frac{\partial g_{k}\left(r_{h}\right)}{\partial r_{h}}+\frac{k-2}{2 \omega_{3}} y_{3}^{k-2} n_{3} F_{k}(x, y) \\
& -\frac{y_{3}^{k-2} x_{3}^{\frac{k-2}{2}} g_{k}\left(r_{h}\right) \cosh r_{h}}{\omega_{3} \sinh ^{3} r_{h}} .
\end{aligned}
$$

Since $B_{h}\left(x, R_{h}\right)=B\left(c_{x}\left(R_{h}\right), x_{3} \sin R_{h}\right)$ for $c_{x}\left(R_{h}\right)=x_{0}+x_{1} e_{1}+x_{2} e_{2}+x_{2} \cosh R_{h}$ we infer that

$$
\lim _{R_{h} \rightarrow 0} \frac{x_{3}^{\frac{k-4}{2}}}{\omega_{3} x_{3}^{3} \sinh ^{3} R_{h}} \int_{\partial B_{h}\left(x, R_{h}\right)} \sinh R_{h} y_{3}^{k-2} \frac{\partial g_{k}}{\partial r_{h}}\left(R_{h}\right) d \sigma_{(k)}=0 .
$$

Similarly, we compute that

$$
\lim _{R_{h} \rightarrow 0} \frac{(k-2) x_{3}^{\frac{k-6}{2}}}{2 \omega_{3} x_{3}^{3} \sinh ^{3} R_{h}} \int_{\partial B_{h}\left(x, R_{h}\right)} y_{3}^{k-2}\left(y_{3}-x_{3} \cosh R_{h}\right) g_{k}\left(R_{h}\right) d \sigma_{(k)}=0 .
$$

Finally, manipulating the last integral, we obtain

$$
\begin{aligned}
& \lim _{R_{h} \rightarrow 0}-\frac{g_{k}\left(R_{h}\right) \cosh R_{h}}{\omega_{3} \sinh ^{3} R_{h}} \int_{\partial B_{h}\left(x, R_{h}\right)} y_{3}^{k-2} x_{3}^{\frac{k-2}{2}} d \sigma_{(k)} \\
& =\lim _{r_{h} \rightarrow 0}-\frac{x_{3}^{\frac{k+4}{2}} \cosh R_{h} g_{k}\left(R_{h}\right)}{\omega_{3} x_{3}^{3} \sinh ^{3} R_{h}} \int_{\partial B_{h}\left(x, R_{h}\right)} y_{3}^{-\frac{k+4}{2}} d \sigma \\
& =-u(x),
\end{aligned}
$$

completing the proof.

Theorem 7. Let $\Omega \subset \mathbb{H}_{+}$be open and $B_{h}(a, \rho)$ a hyperbolic ball with a center a and the hyperbolic radius $\rho$ satisfying $\overline{B_{h}(a, \rho)} \subset \Omega$. If $u$ is a twice continuously differentiable functions in $\Omega$ and $x \in B_{h}(a, \rho)$ then

$$
\begin{aligned}
u(x)= & \int_{\partial B_{h}(a, \rho)}\left(F_{k}(y, x) \frac{\partial u(y)}{\partial n^{k}}-u(y) \frac{\partial F_{k}(y, x)}{\partial n^{k}}\right) d \sigma_{(k)}(y) \\
& -\int_{B_{h}(a, \rho)} \Delta_{k} u(y) F_{k}(y, x) d m_{k}(y)
\end{aligned}
$$

where $d m_{k}=y_{3}^{-2 k} d x, d \sigma_{(k)}=y_{n}^{-\frac{3 k}{2}} d \sigma$ and the outer normal $\frac{\partial u}{\partial n^{k}}=y_{3}^{\frac{k}{2}} \frac{\partial u}{\partial n_{e}}$.

Proof. Denote $\frac{B_{h}(a, \rho)}{B_{h}\left(x, R_{h}\right)} B$ and pick a hyperbolic ball such that $\overline{B_{h}\left(x, R_{h}\right)} \subset B$. Denote $R=B \backslash \overline{B_{h}\left(x, R_{h}\right)}$. Since $F_{k}$ is $k$-hyperbolic harmonic in $R$, we may apply the 
Green's formula

$$
\int_{R}\left(u \Delta_{k} v-v \Delta_{k} u\right) d m_{k}=\int_{\partial R}\left(u \frac{\partial v}{\partial n^{k}}-v \frac{\partial u}{\partial n^{k}}\right) d \sigma_{(k)}
$$

of the Laplace-Beltrami operator

$$
\Delta_{k}=x_{3}^{k}\left(\Delta-\frac{k}{x_{3}} \frac{\partial}{\partial x_{3}}\right)
$$

with respect to the Riemannian metric $d s_{k}^{2}$ (see [1]) and obtain

$$
\begin{aligned}
\int_{R} F_{k}(y, x) \Delta_{k} u d x_{k}= & \int_{\partial B}\left(F_{k}(y, x) \frac{\partial u}{\partial n^{k}}-u \frac{\partial F_{k}(y, x)}{\partial n^{k}}\right) d \sigma(k) \\
& -\int_{\partial B_{h}\left(x, R_{h}\right)}\left(F_{k}(y, x) \frac{\partial u}{\partial n^{k}}-u \frac{\partial F_{k}(y, x)}{\partial n^{k}}\right) d \sigma_{(k)} .
\end{aligned}
$$

Since $\frac{\partial u}{\partial n^{k}}$ and $y_{3}^{-\frac{2 k+2}{2}} x_{3}^{\frac{k-2}{2}} g_{k}\left(d_{h}(x, y)\right)$ are bounded we obtain

$$
\int_{\partial B_{h}\left(x, R_{h}\right)}\left|F_{k}(y, x) \frac{\partial u}{\partial n^{k}}\right| d \sigma_{(k)}(y) \leq \frac{M}{\sinh ^{2} R} \int_{\partial B_{h}\left(x, R_{h}\right)} d \sigma=M \sinh R_{h}
$$

and therefore

$$
\lim _{R_{h} \rightarrow 0} \int_{\partial B_{h}\left(x, R_{h}\right)}\left|F_{k}(y, x) \frac{\partial u}{\partial n^{k}}\right| d \sigma_{(k)}(y)=0 .
$$

Moreover, since $F_{k}(x, y)$ is integrable and $u$ is bounded on $\bar{B}$ we infer

$$
\int_{B_{h}(a, \rho)} \Delta_{k} u(y) F_{k}(y, x) d m_{k}=\lim _{R_{h} \rightarrow 0} \int_{R_{h}} F_{k}(y, x) \Delta_{k} u d m_{k} .
$$

Then applying the previous result we conclude the result.

Using the standard methods, we deduce that

$$
\phi(x)=-\int \Delta_{k} \phi(y) F_{k}(y, x) d m_{k}
$$

for all $\phi \in \mathscr{C}_{0}^{\infty}\left(\mathbb{H}_{+}\right)$. Hence we have reached our main result.

Theorem 8. Let $x$ and $y$ be poins in the upper half space and denote $v=\frac{|k+1|-1}{2}$. The fundamental $k$-hyperbolic harmonic function is 


$$
\begin{aligned}
F_{k}(x, y) & =-\frac{x_{3}^{\frac{k-2}{2}} y_{3}^{\frac{k-2}{2}} Q_{v}^{1}(\lambda(x, y))}{2^{v+1} \omega_{3}\left(\lambda(x, y)^{2}-1\right)^{\frac{1}{2}}} \\
& =\frac{(v+1) x_{3}^{\frac{k-2}{2}} y_{3}^{\frac{k-2}{2}} \int_{0}^{\pi}(\lambda(x, y)+\cos \alpha)^{-v} \sin ^{2 v+1} \alpha d \alpha}{2^{v+1} \omega_{3}\left(\lambda(x, y)^{2}-1\right)} \\
& =\frac{\sqrt{\pi} \Gamma(v+2) x_{3}^{\frac{k-2}{2}} y_{3}^{\frac{k-2}{2}-1} \lambda^{-v}{ }_{2} F_{1}\left(\frac{v}{2}, \frac{v+1}{2} ; \frac{2 v+3}{2} ; \frac{1}{\lambda^{2}}\right)}{2^{v+1} \omega_{3} \Gamma\left(v+\frac{3}{2}\right)\left(\lambda(x, y)^{2}-1\right)} .
\end{aligned}
$$

Corollary 4. Let $x$ and $y$ be points in the upper half-space $\mathbb{H}_{+}$. Then

$$
F_{k}(x, y)=x_{3}^{k+1} y_{3}^{k+1} F_{-k-2}(x, y) .
$$

The previous result follows also from the correspondence principle of Weinstein (see [21]).

Lemma 7. If we denote

$$
K_{k}(f)=\Delta f-\frac{k}{x_{3}} \frac{\partial f}{\partial x_{3}}
$$

then

$$
K_{k}(f)=x_{3}^{k+1} K_{-k-2}\left(x_{3}^{-k-1} f\right) .
$$

A kind of fundamental $k$-hyperbolic harmonic function has also been computed by GowriSankaram and Singman in [13] using more technical deductions. In order to compare the results, we first verify the following lemma.

Lemma 8. Let $\lambda>1$ and $v>-1$. Then

$$
\int_{0}^{\pi}(\lambda-\cos \alpha)^{-v-1} \sin ^{2 v+1} \alpha d \alpha=2^{v+1} Q_{v}^{0}(\lambda)
$$

and therefore

$$
\begin{aligned}
\left(\lambda^{2}-1\right)^{-\frac{1}{2}} Q_{v}^{1}(\lambda) & =-(v+1) 2^{-v-1} \int_{0}^{\pi}(\lambda-\cos \alpha)^{-v-2} \sin ^{2 v+1} \alpha d \alpha \\
& =-2(v+1) x_{2}^{v+2} y_{3}^{v+2} \int_{0}^{\pi}\left(\|x-y\|^{2}+2 x_{3} y_{3}(1-\cos \alpha)\right)^{-v-2} \sin ^{2 v+1} \alpha d \alpha .
\end{aligned}
$$

Proof. Appying [20, S.2.9-4.] and using complex numbers in computations, we obtain

$$
\begin{aligned}
Q_{v}^{0}(\lambda) & =e^{i(v+1) \pi} Q_{v}^{0}(-\lambda)=e^{i(v+1) \pi} 2^{-(v+1)} \int_{0}^{\pi}(-\lambda+\cos \alpha)^{-v-1} \sin ^{2 v+1} \alpha d \alpha \\
& =2^{-(v+1)} \int_{0}^{\pi}(\lambda-\cos \alpha)^{-v-1} \sin ^{2 v+1} \alpha d \alpha
\end{aligned}
$$


Recalling the known formula

$$
Q_{v}^{1}(\lambda)=\left(\lambda^{2}-1\right)^{\frac{1}{2}} \frac{d}{d \lambda} Q_{v}^{0}(\lambda)
$$

we obtain the first equality. The second one follows from it when we substitute $\lambda=\frac{\|x-y\|^{2}+2 x_{3} y_{3}}{2 x_{3} y_{3}}$.

Theorem 9. Let $x$ and $y$ be poins in the upper half space and denote $v=\frac{|k+1|-1}{2}$. The fundamental k-hyperbolic harmonic function is

$$
\begin{aligned}
\omega_{3} F_{k}(x, y) & =\frac{(v+1) x_{3}^{\frac{k-2}{2}} y^{\frac{k-2}{2}} \int_{0}^{\pi}(\lambda-\cos \alpha)^{-v-2} \sin ^{2 v+1} \alpha d \alpha}{2^{v+1}} \\
& =2(v+1) x_{3}^{\frac{k-2}{2}+v+2} y_{3}^{\frac{k-2}{2}+v+2} \int_{0}^{\pi}\left(\|x-y\|^{2}+2 x_{3} y_{3}(1-\cos \alpha)\right)^{-v-2} \sin ^{2 v+1} \alpha d \alpha \\
& =\left\{\begin{array}{cl}
-\frac{k \int_{0}^{\pi}\left(\|x-y\|^{2}+2 x_{3} y_{3}(1-\cos \alpha)\right)^{\frac{k-2}{2}} \sin ^{-k-1} \alpha d \alpha,}{\omega_{3}}, & \text { if } k \leq-1, \\
\frac{(k+2) x_{3}^{k+1} y_{3}^{k+1} \int_{0}^{\pi}\left(\|x-y\|^{2}+2 x_{3} y_{3}(1-\cos \alpha)\right)^{-\frac{k+4}{2}} \sin ^{k+1} \alpha d \alpha}{\omega_{3}}, & \text { if } k \geq-1 .
\end{array}\right.
\end{aligned}
$$

We may compute the following special cases.

1. Let $k=0$. Then

$$
\begin{array}{r}
\phi(x, y)=\frac{1}{2 \omega_{3} x_{3} y_{3}}\left(\frac{1}{\lambda-1}-\frac{1}{\lambda+1}\right) \\
\frac{1}{\omega_{3}}\left(\frac{1}{\|x-y\|^{2}}-\frac{1}{\left\|x-y^{*}\right\|^{2}}\right)
\end{array}
$$

2. Let $k=-2$. Then

$$
\begin{aligned}
F_{-2}(x, y) & =\frac{1}{2 \omega_{3} x_{3}^{2} y_{3}^{2}} \int_{0}^{\pi}\left(\cosh d_{h}(x, y)-\cos \alpha\right)^{-2} \sin \alpha d \alpha \\
& =\frac{1}{2 \omega_{3} x_{3}^{2} y_{3}^{2}}\left(\frac{1}{\lambda-1}-\frac{1}{\lambda+1}\right) \\
& =\frac{1}{\omega_{3} x_{3}^{2} y_{3}^{2}\left(\lambda^{2}-1\right)} \\
& =\frac{1}{2 \omega_{3} x_{3} y_{3}}\left(\frac{1}{\|x-y\|^{2}}-\frac{1}{\left|x-y^{*}\right|^{2}}\right) \\
& =\frac{4}{\omega_{3}\|x-y\|^{2}\left\|x-y^{*}\right\|^{2}} .
\end{aligned}
$$

3. Let $k=2$, then 


$$
\begin{aligned}
2 \omega_{3}^{-1} F_{2}(x, y)= & \int_{0}^{\pi}\left(\cosh d_{h}(x, y)-\cos \alpha\right)^{-3} \sin ^{3} \alpha d \alpha \\
= & {\left[-2^{-1}\left(\cosh d_{h}(x, y)-\cos \alpha\right)^{-2} \sin ^{2} \alpha\right]_{0}^{\pi} } \\
& +\int_{0}^{\pi}\left(\cosh d_{h}(x, y)-\cos \alpha\right)^{-2} \sin \alpha \cos \alpha d \alpha \\
= & -\left[\left(\cosh d_{h}(x, y)-\cos \alpha\right)^{-1} \cos \alpha\right]_{0}^{\pi} \\
& -\int_{0}^{\pi}\left(\cosh d_{h}(x, y)-\cos \alpha\right)^{-1} \sin \alpha d \alpha \\
= & \frac{1}{\lambda-1}+\frac{1}{\lambda+1}-(\log (\lambda+1)-\log (\lambda-1)) \\
= & \frac{2 \lambda}{\lambda^{2}-1}-\log (\lambda+1)+\log (\lambda-1) .
\end{aligned}
$$

Comparing this function with the kernel function computed in [12], we obtain

$$
\begin{aligned}
-\int_{\frac{\|a-x\|}{\left\|x-a^{*}\right\|}}^{1} \frac{\left(1-s^{2}\right)^{2}}{s^{3}} d s & =-\int_{\frac{\|a-x\|}{\left\|x-a^{*}\right\|}}^{1}\left(s^{-3}-2 s^{-1}+s\right) d s \\
& =\frac{\left|x-a^{*}\right|^{2}}{2\|a-x\|^{2}}+2 \log \frac{\|a-x\|}{\left\|x-a^{*}\right\|}-\frac{1}{2} \frac{\|a-x\|^{2}}{\left\|x-a^{*}\right\|^{2}} .
\end{aligned}
$$

Applying the properties (1) and (2), we infer that

$$
-\frac{1}{4} \int_{\|x-x\|}^{1} \frac{\left(1-s^{2}\right)^{2}}{s^{3}} d s=\frac{\lambda}{\lambda^{2}-1}-\frac{\log (\lambda+1)}{2}+\frac{\log (\lambda-1)}{2}
$$

In order to compute the kernel function for $k$-hyperregular functions, we need the following lemma (see [12]).

Lemma 9. If $a \in \mathbb{R}_{+}^{n+1}$ and $c_{a}\left(d_{h}(x, a)\right)=a_{0}+a_{1} e_{1}+a_{2} e_{2}+a_{3} \cosh d_{h}(x, a) e_{3}$ then

$$
\bar{D}^{x} \lambda(x, a)=\frac{\overline{x-c_{a}\left(d_{h}(x, a)\right)}}{x_{3} a_{3}} .
$$

Theorem 10. Denote $r_{h}=d_{h}(x, y), t=\frac{k-2}{2}, v=\frac{|k+1|-1}{2}$ and define as earlier

$$
g_{k}\left(d_{h}(x, y)\right)=\frac{|k+1|+1}{2^{v+2}} \int_{0}^{\pi}\left(\cosh d_{h}(x, y)+\cos \alpha\right)^{-v} \sin ^{2 v+1} \alpha d \alpha .
$$

The k-hyperregular kernel is the function 


$$
\begin{aligned}
h_{k}(x, y) & =\bar{D}^{x}\left(F_{k}(x, y)\right) \\
& =-x_{3}^{\frac{k-2}{2}} y_{3}^{\frac{k-2}{2}}\left(\frac{\operatorname{tg}\left(r_{h}\right) e_{3}}{2 x_{3} \sinh ^{2} r_{h}}+\left(\frac{\sinh r_{h} g^{\prime}\left(r_{h}\right)-2 g\left(r_{h}\right) \cosh r_{h}}{2 \sinh ^{4} r_{h}}\right) \frac{\overline{x-c_{y}\left(r_{h}\right)}}{x_{3} y_{3}}\right) \\
& =x_{3}^{\frac{k-2}{2}} y_{3}^{\frac{k+4}{2}} w_{k}(x, y) p(x, y) \\
& =x_{3}^{\frac{k-2}{2}} y_{3}^{\frac{k+4}{2}} p(x, y) v_{k}(x, y)
\end{aligned}
$$

where

$$
w_{k}(x, y)=-t e_{3} g_{k}\left(r_{h}\right) \frac{x-P y}{y_{3}}+\sinh r_{h} g_{k}^{\prime}\left(r_{h}\right)-(t+2) g_{k}\left(r_{h}\right) \cosh r_{h}
$$

and

$$
p(x, y)=\frac{\left(x-c_{y}\left(r_{h}\right)\right)^{-1}}{2 x_{3}\left\|x-c_{y}\left(r_{h}\right)\right\|^{2}}
$$

is 2-hyperregular with respect to $x$.

Proof. The function $F_{k}(x, y)$ is $k$-hyperbolic and therefore the function $h_{k}=\bar{D}^{x} F_{k}(x, y)$ is $k$-hyperregular outside $y$ and $y^{*}$. Denoting $t=\frac{k-2}{2}$ and $\lambda(x, y)=\cosh r_{h}$, we compute as follows

$$
\frac{h_{k}(x, y)}{x_{3}^{\frac{k-2}{2}} y_{3}^{\frac{k+4}{2}}}=-\frac{t e_{3} g\left(r_{h}\right)}{2 x_{3} y_{3}^{3} \sinh ^{2} r_{h}}+\left(\frac{\sinh r_{h} g^{\prime}\left(r_{h}\right)-2 g\left(r_{h}\right) \cosh r_{h}}{2 y_{3}^{3} \sinh ^{3} r_{h}}\right) \bar{D}^{x} r_{h} .
$$

Applying [12] we obtain

$$
\bar{D}^{x} r_{h}=\frac{\overline{x-c_{y}\left(r_{h}\right)}}{x_{3} y_{3} \sinh r_{h}}
$$

and

$$
\frac{x_{3} \bar{D}^{x} r_{h}}{y_{3}^{3} \sinh ^{3} r_{h}}=\frac{\overline{x-c_{y}\left(r_{h}\right)}}{\left\|x-c_{y}\left(r_{h}\right)\right\|^{4}}=\frac{\left(x-c_{y}\left(r_{h}\right)\right)^{-1}}{\left\|x-c_{y}\left(r_{h}\right)\right\|^{2}} .
$$

Since

$$
\begin{aligned}
\frac{x-c_{y}\left(r_{h}\right)}{x_{3} y_{3}} \frac{\left(x-c_{y}\left(r_{h}\right)\right)^{-1}}{\left\|x-c_{y}\left(r_{h}\right)\right\|^{2}} & =\frac{1}{x_{3} y_{3}\left\|x-c_{y}\left(r_{h}\right)\right\|^{2}} \\
& =\frac{1}{x_{3} y_{3}^{3} \sinh ^{2} r_{h}} .
\end{aligned}
$$

Hence we obtain

$$
\frac{h_{k}(x, y)}{y_{3}^{t+3} x_{3}^{t}}=w_{k}(x, y) \frac{\left(x-c_{y}\left(r_{h}\right)\right)^{-1}}{2 x_{3}\left\|x-c_{y}\left(r_{h}\right)\right\|^{2}}
$$

where

$$
w_{k}(x, y)=-t e_{3} g_{k}\left(r_{h}\right) \frac{x-P y}{y_{3}}+\sinh r_{h} g_{k}^{\prime}\left(r_{h}\right)-(t+2) g_{k}\left(r_{h}\right) \cosh r_{h} .
$$


Using the similar deductions as in [4] we may prove the formula for $S$ and $T$ parts.

Theorem 11. Let $\Omega$ and be an open subsets of $\mathbb{H}_{+}$(or $\mathbb{H}_{\text {.- }}$ ). Assume that $K$ is an open subset of $\Omega$ and $\bar{K} \subset \Omega$ is a compact set with the smooth boundary whose outer unit normal field is denoted by $v$. If $f$ is $k$-hyperregular in $\Omega$ and $a \in K$, then

$$
\begin{aligned}
S f(a) & =-\frac{1}{2} \int_{\partial K} S\left(h_{k}(y, a) v f(y)+f(y) v h_{k}(y, a)\right) \frac{d \sigma}{y_{3}^{k}} \\
& =\frac{1}{2} \int_{\partial K} S\left[h_{k}(y, a), \overline{v(y)}, f(y)\right] \frac{d \sigma}{y_{3}^{k}}-\frac{1}{2} \int_{\partial K} S h_{k}(y, a)\langle\overline{v(y)}, f(y)\rangle \frac{d \sigma}{y_{3}^{k}} .
\end{aligned}
$$

Proof. Let $a \in K$. Denote $R=K \backslash B_{h}\left(a, r_{h}\right)$ and

$$
A=\int_{\partial K} S\left(h_{k}(y, a) v f(y)+f(y) v h_{k}(y, a)\right) \frac{d \sigma}{y_{3}^{k}} .
$$

Then we obtain

$$
\begin{aligned}
0 & =\int_{\partial R} S\left(h_{k}(y, a) v f(y)+f(y) v h_{k}(y, a)\right) \frac{d \sigma}{y_{3}^{k}} \\
& =A-\int_{\partial B_{h}\left(a, r_{h}\right)} S\left(h_{k}(y, a) v(y) f(y)+f(y) v(y) h_{k}(y, a)\right) \frac{d \sigma}{y_{3}^{k}} .
\end{aligned}
$$

By virtue of Proposition 1, we deduce that

$$
v(y)=\frac{y-c_{a}\left(r_{h}\right)}{\left\|y-c_{a}\left(r_{h}\right)\right\|} .
$$

Hence we obtain

$$
\begin{aligned}
A & =-\lim _{r_{h}} \frac{a_{3}^{\frac{k-4}{2}}}{2 \omega_{3}\left\|a-c_{a}\left(r_{h}\right)\right\|^{3}} \int_{\partial B_{h}\left(a, r_{h}\right)} S\left(w_{k}(y, a) f(y)+f(y) v_{k}(y, a)\right) \frac{d \sigma}{y_{3}^{\frac{k-4}{2}}} \\
& =-2 f(a)
\end{aligned}
$$

The last formula follows from (7) and the definition of the triple product.

Similarly we may verify the result for the $T$-part. The main difference is that we use the surface measure $d \sigma$, not $y_{3} d \sigma$.

Theorem 12. Let $\Omega$ be an open subsets of $\mathbb{H}_{+}$(or $\mathbb{H}_{-_{-}}$). Assume that $K$ is an open subset of $\Omega$ and $\bar{K} \subset \Omega$ is a compact set with the smooth boundary whose outer unit normal field at $y$ is denoted by $v$. If $f$ is $k$-hyperregular in $\Omega$ and $a \in K$, 


$$
\begin{aligned}
T f(a) & =-\frac{a_{3}^{k}}{2} \int_{\partial K} T\left(h_{-k}(y, a) v(y) f(y)+f(y) v(y) h_{-k}(y, a)\right) d \sigma \\
& =\frac{a_{3}^{k}}{2}\left(\int_{\partial K} T\left[h_{-k}(y, a), \overline{v(y)}, f(y)\right] d \sigma-\int_{\partial K} T h_{-k}(y, a)\langle\overline{v(y)}, f(y)\rangle d \sigma\right) .
\end{aligned}
$$

\section{Conclusion}

Our main results produce integral formulas for the $T$ - and $S$-parts of $k$-hyperregular functions. An interesting problem is to research integral operators produced by these formulas. However, these results requires much computations and therefore they are left to the consequent publications.

Acknowledgements The second author wishes to thank YTK for financial support to complete this job.

\section{References}

1. Akin, Ö. and H. Leutwiler, On the invariance of the solutions of the Weinstein equation under Möbius transformations, Classical and modern hypercomplextential theory and applications (Chateau de Bonas, 1993), 19 - 29, NATO Adv. Sci. Inst. Ser. C Math. Phys. Sci., 430, Kluwer, Dordrecht, 1994.

2. Andrews, G., R. Askey and R. Roy, Special functions, Encyclopedia of Mathematics and Applications 71, Cambridge University Press, 1999.

3. Eriksson-Bique, S.-L., k-hypermonogenic functions, In Progress in analysis: H. Begerh, R. Gilbert, and M. W. Wong, eds, World Scientific, Singabore, 2003, 337-348.

4. Eriksson, S.-L., Integral formulas for hypermonogenic functions, Bull. of the Belgian Math. Soc. 11 (2004), 705-717.

5. Eriksson, S.-L., Hyperbolic Extensions of Integral Formulas, Adv. appl. Clifford alg. 20, Numbers 3-4, (2010), 575-586.

6. Eriksson, S.-L., Integral formulas for hypermonogenic functions, Bull. of the Belgian Math. Soc. 11 (2004), 705-717.

7. Eriksson-Bique, S.-L. and H. Leutwiler, Hypermonogenic functions, In Clifford Algebras and their Applications in Mathematical Physis, Vol. 2, Birkhäuser, Boston, 2000, 287-302.

8. Eriksson, S.-L. and H. Leutwiler, Hyperbolic Function Theory, Adv. appl. Clifford alg. 17 (2007), 437-450.

9. Eriksson, S.-L. and H. Leutwiler, Contributions to the Theory of Hypermonogenic Functions, Complex Variables and Elliptic Equations Vol. 51, Nos 5-6, (2006),547-561.

10. Eriksson, S-L. and H. Leutwiler, Hyperbolic harmonic functions and their function theory, in Potential theory and Stochastics in Albac, Theta 2009, 85-100.

11. Eriksson, S.-L. and H. Orelma, General Integral Formulas for k-hyper-mono-genic Functions, Adv. Appl. Clifford Algebras Volume 27, Issue 1, (2017), 99-110.

12. Eriksson, S.-L. and H. Orelma, On Hypermonogenic Functions, Complex Variables and Elliptic Equations: An International Journal, Volume 58, Issue 7, (2013), 975-990.

13. GowriSankaram, K. and D. Singman, Littlewood theorem for Weinstein potentials, Illinois Journal of Math., Volume 41 (4), 1997. 
14. Gürlebeck, K., K. Habetha, and W. Sprößig, Holomorphic Functions in the Plane and ndimensional Space, Birkhäuser, Basel, 2008.

15. Hempfling, Th. and H. Leutwiler, Modified Quaternionic Analysis in $\mathbb{R}^{4}$, In Clifford Algebras and Their Application in Mathematical Physics, Fundamental Theories of Physics 94, pp 227237.

16. Leutwiler, H., Best constants in the Harnack inequality for the Weinstein equation, Aequationes Math. 34, no. 2 - 3, (1987), $304-315$.

17. Leutwiler, H., Modified Clifford analysis, Complex Variables 17 (1992), 153-171.

18. Leutwiler, H., Appendix: Lecture notes of the course "Hyperbolic harmonic functions and their function theory". Clifford algebras and potential theory, 85-109, Univ. Joensuu Dept. Math. Rep. Ser., 7, Univ. Joensuu, Joensuu, 2004.

19. Orelma, H., New Perspectives in Hyperbolic Function Theory, TUT, Dissertation, 892, 2010.

20. Polyanin, A. and F. Zaitsev, Handbook of Exact Solutions for Ordinary Differential Equations, Second Edition, A CRC Press Company, Boca Raton, London, New York Washington, D.C. 2003.

21. Weinstein, A., Generalized axially symmetric potential theory, Bull. Amer. Math. Soc. 69 (1953), 20-38. 